\title{
ON THE LIFTING PROPERTY. III ${ }^{1}$
}

\section{BY A. IONESCU TULCEA AND C. IONESCU TULCEA}

Communicated by Walter Rudin, September 9, 1963

1. Let $Z$ be a locally compact space and $\mu \neq 0$ a positive Radon measure on $Z$. Let $M_{R}^{\infty}(Z, \mu)$ be the Banach algebra of all bounded real-valued $\mu$-measurable functions defined on $Z$, endowed with the $\operatorname{norm} f \rightarrow\|f\|_{\infty}=\sup _{z \in Z}|f(z)|$. Let $C_{R}^{\infty}(Z)$ be the subalgebra of $M_{R}^{\infty}(Z, \mu)$ consisting of all bounded continuous functions on $Z$ and $\Re(Z)$ the subalgebra of all $f \in C_{R}^{\infty}(Z)$ having compact support. For two functions $f$ and $g$ defined on $Z$ we shall write $f \equiv g$ whenever $f$ and $g$ coincide locally almost everywhere.

Let now $T: f \rightarrow T_{f}$ be a mapping of $M_{R}^{\infty}(Z, \mu)$ into $M_{R}^{\infty}(Z, \mu)$. Properties of $T$ such as those listed below will be considered in what follows:

(I) $T_{f} \equiv f$;

(II) $f \equiv g$ implies $T_{f}=T_{g}$;

(III) $T_{1}=1$;

(IV) $f \geqq 0$ implies $T_{f} \geqq 0$;

(V) $T_{\alpha f+\beta g}=\alpha T_{f}+\beta T_{g}$;

(VI) $T_{f g}=T_{f} T_{g}$;

(VII) $T_{f}=f$ if $f \in C_{R}^{\infty}(Z)$.

A mapping $T: f \rightarrow T_{f}$ of $M_{R}^{\infty}(Z, \mu)$ into $M_{R}^{\infty}(Z, \mu)$ satisfying (I)-(V) will be called a linear lifting of $M_{R}^{\infty}(Z, \mu)$; if the condition (VI) is also verified the mapping will be called a lifting of $M_{R}^{\infty}(Z, \mu)$. A strong linear lifting [strong lifting] of $M_{R}^{\infty}(Z, \mu)$ is a linear lifting [lifting] which verifies also (VII).

If $T: f \rightarrow T_{f}$ is a lifting of $M_{R}^{\infty}(Z, \mu)$ and $A$ is a $\mu$-measurable set then we shall denote by $\rho_{T}(A)$ the set defined by the equation ${ }^{2}$ $T_{\phi_{A}}=\phi_{\rho_{T}(A)}$. For each $z \in Z$ denote by $\vartheta_{T}(z)$ the set of all parts $\rho_{T}(V)$ where $V$ belongs to ${ }^{3} \vartheta(z)$ and is $\mu$-measurable. If $\tau_{T}$ is the set of all parts $\rho_{T}(A)-N$ where $A$ is $\mu$-measurable and $N$ is locally $\mu$ negligible then $\tau_{T}$ is a topology on $Z$ (this result is essentially due to $\mathrm{J}$. Oxtoby and has been given by him in a lecture at Yale in the fall of 1960).

THEOREM 1. Let $T: f \rightarrow T_{f}$ be a lifting of $M_{R}^{\infty}(Z, \mu)$. Then the following assertions are equivalent: (1.1) $T$ is a strong lifting; (1.2) There is

\footnotetext{
${ }^{1}$ Research supported by the U. S. Army Research Office (Durham) under contract DA-ARO(D)-31-124-G218.

${ }^{2}$ For $X \subset Z, \phi_{X}$ denotes the characteristic function of $X$.

${ }^{3}$ For each $z \in Z, \mathcal{V}(z)$ denotes the set of all neighborhoods of $z$.
} 
$V \subset M_{R}^{\infty}(Z, \mu)$, dense in $\mathfrak{K}(Z)$ for the topology induced by the norm $f \rightarrow\|f\|_{\infty}$, such that $T_{f}=f$ for all $f \in V ;(1.3) \rho_{T}(U) \supset U$ for all $U \subset Z$ open; (1.4) $\rho_{T}(F) \subset F$ for all $F \subset Z$ closed; (1.5) $\tau_{T}$ is stronger than the topology of $Z ;(1.6)$ For each $z \in Z, V_{T}(z)$ is a fundamental system of neighborhoods of $z$.

Let $M_{R}(Z, \mu)$ be the algebra of all locally bounded (that is bounded on every compact) real-valued $\mu$-measurable functions defined on $Z$. By $C_{R}(Z)$ we shall denote the subalgebra or all real-valued continuous functions on $Z$. A mapping $T: f \rightarrow T_{f}$ of $M_{R}(Z, \mu)$ into $M_{R}(Z, \mu)$ which verifies conditions (I)-(VI) will be called a lifting of $M_{R}(Z, \mu)$. A strong lifting of $M_{R}(Z, \mu)$ is a lifting $T: f \rightarrow T_{f}$ such that $T_{f}=f$ for all $f \in C_{R}(Z)$.

THEOREM 2. The following conditions are equivalent: (2.1) There is a strong linear lifting of $M_{R}^{\infty}(Z, \mu) ;(2.2)$ There is a strong lifting of $M_{R}^{\infty}(Z, \mu) ;(2.3)$ There is a strong lifting of $M_{R}(Z, \mu)$.

REMARK. There is a compact space $Z \neq \varnothing$, a positive Radon measure $\mu$ on $Z$ with Supp $\mu=Z$ and a lifting $T: f \rightarrow T_{f}$ of $M_{R}^{\infty}(Z, \mu)$ such that

$$
Z=\bigcup_{f \in C_{R}(z)}\left\{z \mid T_{f}(z) \neq f(z)\right\} .
$$

2. For a locally compact space $B$ and a positive Radon measure $\alpha$ on $B$ we shall denote by $\mathrm{e}(B, \alpha)$ the set of all locally countable families $\left(K_{j}\right)_{j \in J}$ of disjoint compact parts of $B$ such that the complement of $\bigcup_{j \in J} K_{j}$ is locally $\alpha$-negligible.

Let now $Z$ and $B$ be two locally compact spaces and $\alpha$ a positive Radon measure on $B$. For each mapping $\lambda: b \rightarrow \lambda_{b}$ of $B$ into $\mathfrak{N}_{+}(Z)$ and each $g \in \mathfrak{K}(Z)$ we shall denote by $\langle g, \lambda\rangle$ the mapping $b \rightarrow\left\langle g, \lambda_{b}\right\rangle$. Suppose now $\alpha \neq 0$ and let $T: f \rightarrow T_{f}$ be a lifting of $M_{R}^{\infty}(B, \alpha)$; to shorten the notation we shall sometimes write $\rho_{T}(f)$ instead of $T_{f}$, for $f \in M_{R}^{\infty}(B, \alpha)$. If $\lambda: b \rightarrow \lambda_{b}$ is a mapping of $B$ into $\mathfrak{N}_{+}(Z)$ we shall write

$$
\rho_{T}[\lambda]=\lambda
$$

whenever there is $\left(K_{j}\right)_{j \in J} \in \mathcal{C}(B, \alpha)$ with the following properties:

(1) $\phi_{K_{j}}\langle g, \lambda\rangle \in M_{R}^{\infty}(B, \alpha)$ for every $j \in J$ and $g \in \Re(Z)$;

(2) $\rho_{T}\left(\phi_{K_{j}}\langle g, \lambda\rangle\right)=\phi_{\rho_{T}\left(K_{j}\right)}\langle g, \lambda\rangle$ for every $j \in J$ and $g \in \mathscr{K}(Z)$.

A mapping $\lambda: b \rightarrow \lambda_{b}$ of $B$ into $\Re_{+}(Z)$ will be called appropriate with respect to $(\alpha, T)$ if:

$4 \mathfrak{M}(Z)$ is the vector space of all Radon measures on $Z$ and $\mathfrak{N}_{+}(Z)$ the cone of all positive Radon measures on $Z$. 
(3) $\rho_{T}[\lambda]=\lambda$;

(4) $\langle g, \lambda\rangle$ is essentially $\alpha$-integrable for each $g \in \mathfrak{K}(Z)$ (that is, $\lambda$ is scalarly essentially $\alpha$-integrable if $\operatorname{Tr}(Z)$ is endowed with the topology $\sigma(\mathfrak{T}(Z), \mathfrak{K}(Z)))$.

Let $Z$ and $B$ be two locally compact spaces and $\mu$ a positive Radon measure on $Z$. Recall that a mapping $p: Z \rightarrow B$ is $\mu$-proper if it is $\mu$-measurable and if $f \circ p$ is essentially $\mu$-integrable for each $f \in \mathcal{K}(B)$. The Radon measure $p(\mu)$ is then defined by the equations $\langle f, p(\mu)\rangle$ $=\int_{B} f \circ p d \mu, f \in \mathfrak{K}(B)$. If $\alpha$ is a positive Radon measure on $B$ and $\psi: B \rightarrow R$ a locally $\alpha$-integrable function then $\psi \cdot \alpha$ is the Radon measure defined by the equations $\langle f, \psi \cdot \alpha\rangle=\int_{B} f \psi d \alpha, f \in \mathfrak{K}(B)$. For the above and for other definitions concerning integration see [1].

THEOREM 3. Let $Z$ and $B$ be two locally compact spaces, $\mu$ a positive Radon measure on $Z, p$ a $\mu$-proper mapping of $Z$ into $B$ and $\nu=p(\mu)$. Let now $\alpha$ be a positive Radon measure on $B$ such that $\nu=\psi \cdot \alpha$ for some locally $\alpha$-integrable function $\psi$. Suppose $\alpha \neq 0$ and let $T: f \rightarrow T_{f}$ be $a$ lifting of $M_{R}^{\infty}(B, \alpha)$. Then:

(3.1) There is a mapping $\lambda: b \rightarrow \lambda_{b}$ of $B$ into $\mathfrak{N}_{+}(Z)$, appropriate with respect to $(\alpha, T)$ such that:

(5) $\left\|\lambda_{b}\right\|=\psi(b)$ locally almost everywhere for $\alpha$;

(6) $\int_{Z}(f \circ p) g d \mu=\int_{B} f(b)\left\langle g, \lambda_{b}\right\rangle d \alpha(b)$ for every $f \in \mathcal{K}(B)$ and $g \in \mathcal{K}(Z) .^{5}$

(3.2) Moreover, if $T$ is a strong lifting then $\lambda_{b}$ is concentrated on $p^{-1}(\{b\})$ locally almost everywhere for $\alpha$.

(3.3) Let $\lambda^{\prime}: b \rightarrow \lambda_{b}^{\prime}$ and $\lambda^{\prime \prime}: b \rightarrow \lambda_{b}^{\prime \prime}$ be two mappings of $B$ into $\mathfrak{M}_{+}(Z)$, appropriate with respect to $(\alpha, T)^{6}$ and such that:

(7) $\lambda_{b}^{\prime}$ and $\lambda_{b}^{\prime \prime}$ are concentrated on $p^{-1}(\{b\})$ locally almost everywhere for $\alpha$;

(8) $\mu=\int_{B} \lambda_{b}^{\prime} d \alpha(b)=\int_{B} \lambda_{b}^{\prime \prime} d \alpha(b)$.

Then $\lambda_{b}^{\prime}=\lambda_{b}^{\prime}$ locally almost everywhere for $\alpha$.

The next result is in a certain sense converse to Theorem 3:

Theorem 4. Let $B$ be a locally compact space, $\alpha \neq 0$ a positive Radon measure on $B$ with Supp $\alpha=B$ and $T: f \rightarrow T_{f}$ a lifting of $M_{R}^{\infty}(B, \alpha)$. Then the assertions (4.1) and (4.2) below are equivalent:

(4.1) There is a locally $\alpha$-negligible set $B_{\infty} \subset B$ such that $T_{f}(b)=f(b)$ for each $f \in C_{R}^{\infty}(B)$ and $b \in B_{\infty}$;

(4.2) For every locally compact space $Z$, positive Radon measure $\mu$ on $Z$ and $\mu$-proper mapping $p$ of $Z$ into $B$ such that $\nu=p(\mu)$ is absolutely continuous with respect to $\alpha$, there is a mapping $\lambda: b \rightarrow \lambda_{b}$ of $B$ $\alpha)$.

${ }^{\sigma}$ From (6) we deduce that $\mu=\int_{B} \lambda_{b} d \alpha(b)$ (i.e. $\mu$ is the integral of $\lambda$ with respect to

6 Here the lifting $T$ is not necessarily supposed to be strong. 
into $\mathfrak{T}_{+}(Z)$, appropriate with respect to $(\alpha, T)$ and having the properties:

(9) $\mu=\int_{B} \lambda_{b} d \alpha(b)$;

(10) $\lambda_{b}$ is concentrated on $p^{-1}(\{b\})$ locally almost everywhere for $\alpha$.

REMARK. If $T$ is a lifting having the property stated in (4.1) then there is a strong lifting $T^{\prime}$ of $M_{R}^{\infty}(B, \alpha)$ such that $T_{f}^{\prime}(b)=T_{f}(b)$ for all $f \in M_{R}^{\infty}(B, \alpha)$ and $b \in B_{\infty}$.

3. Let $Z$ be a locally compact space and $\mu$ a positive Radon measure on $Z$. To simplify some of the following statements we shall say that $(Z, \mu)$ has the strong lifting property whenever there is a strong lifting of $M_{R}^{\infty}(Z, \mu)$.

In the statements below $Z \neq \varnothing$ is a locally compact space and $\mu$ a positive Radon measure on $Z$ with Supp $\mu=Z$.

(A) The couple $(Z, \mu)$ has the strong lifting property in each of the following cases: (i) $Z$ is metrizable; (ii) $(Z, \mu)$ is hyperstonean (that is $Z$ is stonean and every rare set is locally $\mu$-negligible); (iii) $\mu$ is atomic.

(B) If $(Z, \mu)$ has the strong lifting property and $K \subset Z, K \neq \varnothing$ is a compact such that Supp $\mu_{K}=K$ then $\left(K, \mu_{K}\right)$ has the strong lifting property.

(C) If $\left(K_{j}\right)_{j \in J} \in \mathfrak{e}(Z, \mu)$ is such that $\left(K_{j}, \mu_{K_{j}}\right)$ has the strong lifting property for each $j \in J$ then $(Z, \mu)$ has the strong lifting property.

(D) Let $Z_{1}, Z_{2}$ be two locally compact spaces and $\mu_{1}, \mu_{2} \neq 0$ two positive Radon measures on $Z_{1}, Z_{2}$ respectively. Suppose that $\left(Z_{1}, \mu_{1}\right)$ has the strong lifting property, $Z_{2}$ is metrizable and Supp $\mu_{2}=Z_{2}$. Then $\left(Z_{1} \times Z_{2}, \mu_{1} \otimes \mu_{2}\right)$ has the strong lifting property.

(E) Let $\left(Z_{j}\right)_{j \in J}$ be a family of metrizable compact spaces and for each $j \in J$ let $\mu_{j}$ be a positive Radon measure on $Z_{j}$ with $\mu_{j}\left(Z_{j}\right)=1$ and Supp $\mu_{j}=Z_{j}$; let $Z_{\infty}=\prod_{j \in J} Z_{j}$ and $\mu_{\infty}=\otimes_{j \in J} \mu_{j}$. Then the couple $\left(Z_{\infty}, \mu_{\infty}\right)$ has the strong lifting property.

Denote by $C_{R}^{\infty}(R,+)\left[C_{R}^{\infty}(R,-)\right]$ the algebra of all bounded realvalued functions, defined on the real line $R$, continuous on the right [continuous on the left]. Let $\mu$ be the Lebesgue measure on $R$.

THEOREM 5. There is a lifting $T: f \rightarrow T_{f}$ of $M_{R}^{\infty}(R, \mu)$ such that $T_{f}=f$ for every $f \in C_{R}^{\infty}(R,+)\left[f \in C_{R}^{\infty}(R,-)\right]$.

4. Remarks. (1) Theorem 1 in [1, Chap. 6, pp. 58-63], some of the results in [2] (see also [4]) and the result in [3, §6, pp. 82-84] are particular cases of Theorem 3 above. Theorem 2 in [1, Chap. 6, pp. 64-65] can be also generalized using the strong lifting. For certain other methods and results concerning the disintegration of measures see also [7]. (2) The result in (E) is essentially contained in [6]. (3) It is known that for every $(Z, \mu), \mu \neq 0$, there is a lifting 
of $M_{R}^{\infty}(Z, \mu)$ (see [5] and [6]).

Problem. Decide whether or not every couple $(Z, \mu)$, with $\mu \neq 0$ and Supp $\mu=Z$, has the strong lifting property.

\section{BIBLIOGRAPHX}

1. N. Bourbaki, Integration, Chaps. I-VI, Hermann, Paris, 1952-1960.

2. J. Dieudonné, Sur le theorème de Lebesgue-Nykodim. III, Ann. Univ. Grenoble Sect. Sci. Math. Phys. (N.S.) 23 (1948), 25-53.

3. - Sur le théorème de Lebesgue-Nykodim. IV, J. Indian Math. Soc. (N.S.) 15 (1951), 77-86.

4. P. R. Halmos, On a theorem of Dieudonne, Proc. Nat. Acad. Sci. U.S.A. 35 (1939), 38-42.

5. A. Ionescu Tulcea and C. Ionescu Tulcea, On the lifting property. I, J. Math. Anal. Appl. 3 (1961), 537-546.

6. D. Maharam, On a theorem of von Neumann, Proc. Amer. Math. Soc. 9 (1958), 987-994.

7. G. Mokobodzki, Barycentres gênéralisés, Séminaire Brelot-Choquet-Deny, Faculté des Sciences de Paris, Juin, 1962.

University of Pennsylvania 